\title{
Influence des diffuseurs aubés sur le fonctionnement des pompes centrifuges
}

\author{
The influence of bladed diffusers on a centrifugal pump working
}

\author{
par Michel Toussaint \\ Conservatoire National des Arts et Métiers Paris
}

François Hureau

C E T I M Nantes

Jean-François Lapray

GEC Alsthom ACB Bergeron

Flow inside centrifugal turbomachines is always turbulent and presents unsteady flow characteristics due to the interaction between fixed and rotating parts. Moreover, during off-peak running, the flow angle/blade profile mismatch causes large local speed fluctuations which accentuate and amplify turbulence and unsteady flow levels particularly in rotor-stator interaction zones. Measurements of typical turbulence parameters within the rotor are only possible by means of such techniques as the laser velocimeter. A comparative analysis of flow in two pump scale models is presented in this paper. Each pump comprises both a smooth and bladed nozzle, and averaged and fluctuating speed vector and turbulence values are presented and analysed.

\section{IINTRODUCTION}

Il est parfaitement établi que, dans une turbomachine, l'interaction mutuelle de deux rangées d'aubages en mouvement de rotation relatif, engendre un écoulement turbulent qui présente des caractéristiques de périodicité et d'instationnarité difficiles à aborder et analyser, tant par le calcul numérique que par l'expérimentation. Des résultats précédents [1] [2] [3] ont montré que l'écoulement délivré par la roue était très peu influencé par la configuration aval (diffuseur lisse ou aubé) et ceci même à débit fortement réduit. Les résultats présentés ici confirment cette tendance et devraient faciliter la conception de machines plus silencieuses sur le plan hydrodynamique des écoulements.

\section{II — INSTALLATIONS EXPÉRIMENTALES}

\subsection{Maquettes d'essais}

Les maquettes des deux pompes sont représentées sur la figure 1. Pour la pompe de Ns32, l'axe de rotation est vertical alors qu'il est horizontal pour celle de Ns20. Dans les deux cas, l'écoulement sortant de la roue est récupéré par un diffuseur (lisse ou aubé), puis collecté dans un caisson circulaire relié à la tuyauterie de refoulement. Ces dispositions assurent l'axisymétrie de l'écoulement en entrée et sortie de roue lors des fonctionnements avec diffuseur lisse. Les deux maquettes sont équipées de hublots en verre permettant des mesures de vitesses par vélocimétrie laser. 


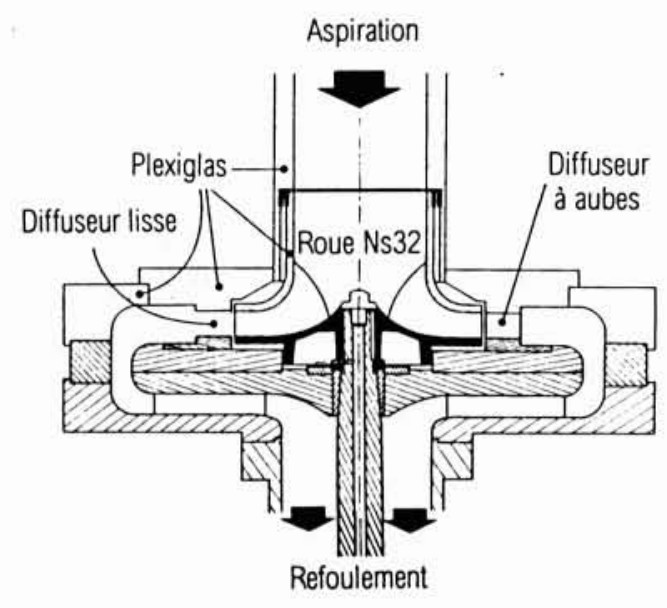

CETIM Nantes Ns 32

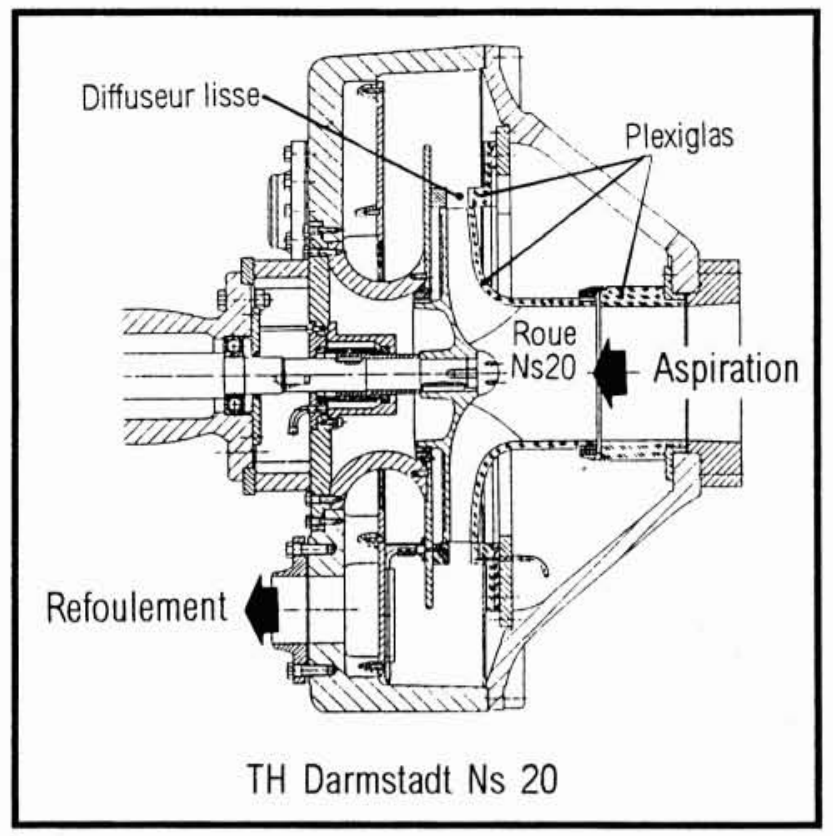

1. Maquettes des deux pompes.

\subsection{Instrumentation}

Les mesures de vitesses et de turbulence ont été réalisées à l'aide de vélocimètres laser à deux réseaux de franges d'interférence schématisés figure 2. L'ensemencement des écoulements est réalisé à l'aide de très fines particules de dioxyde de titane. Chaque mesure permet l'acquisition simultanée de deux composantes du vecteur vitesse absolue $(V d$ et $V u)$ et de la position angulaire de la roue $(\theta)$.

\subsection{Roues et diffuseurs}

Les caractéristiques des deux roues et de leurs diffuseurs sont résumées sur la figure 3 et le tableau 1.

\section{III — RÉSULTATS EXPÉRIMENTAUX}

\subsection{Ecoulement interne dans une turbomachine centri- fuge}

A l'entrée d'une telle turbomachine, l'écoulement turbulent est généralement, dans des conditions optimales d'utilisation, axisymétrique et stationnaire. Dès l'entrée roue cependant, il acquiert un caractère instationnaire périodique, à cause de la présence d'aubages et du mouvement relatif existant entre la roue et le reste de la machine. Les phénomènes visqueux prennent de l'importance et l'écoulement

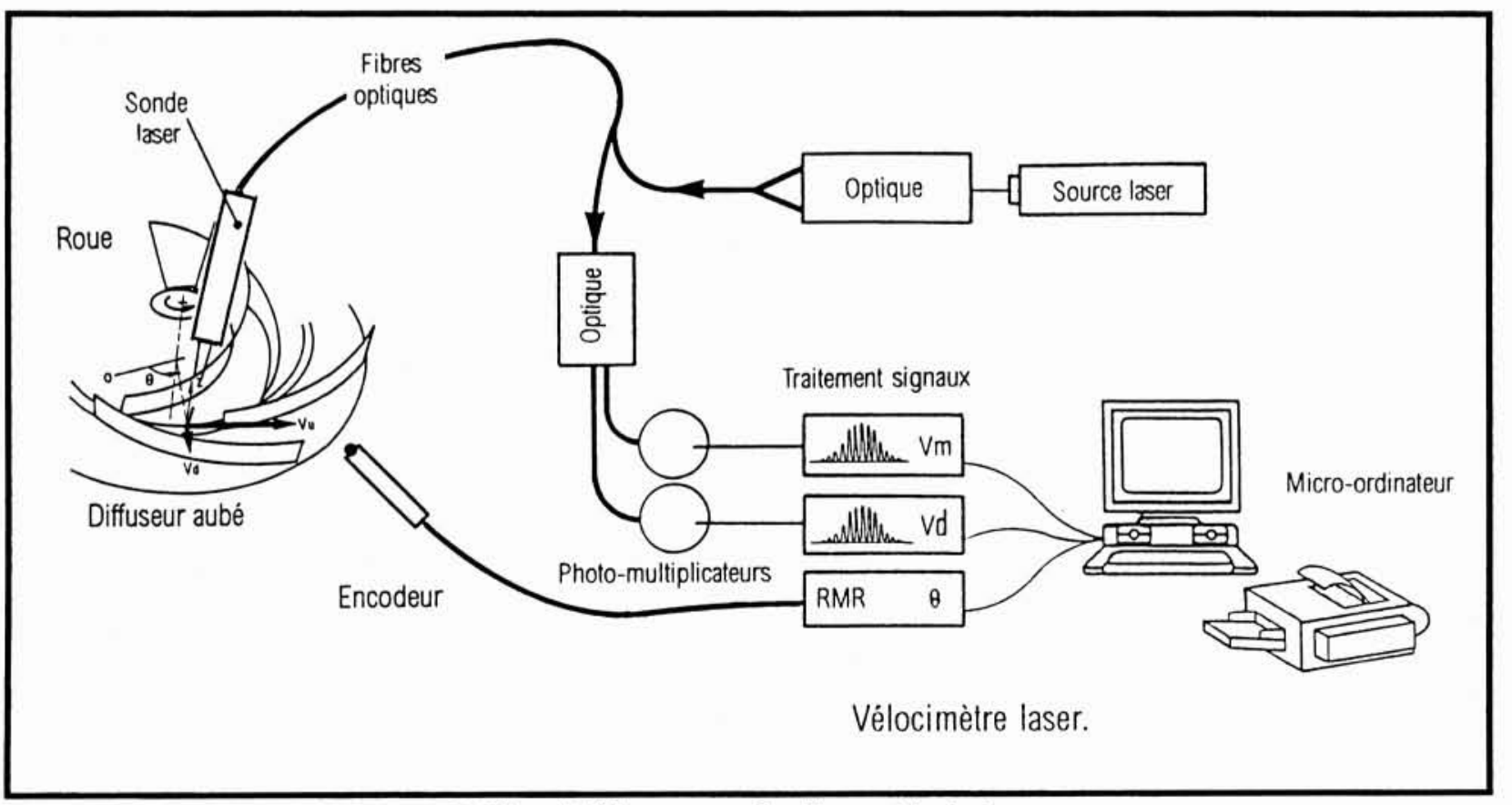

2. Dispositif de mesures de vitesse et turbulence. 


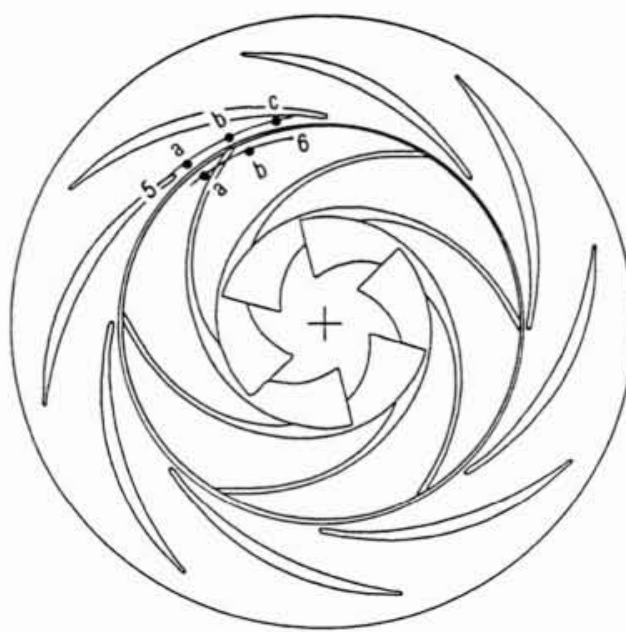

Roue Ns 32

\begin{tabular}{|l|r|c|l|}
\cline { 2 - 3 } \multicolumn{1}{c|}{} & Ns 32 & Ns 20 & \multicolumn{1}{|c}{} \\
\hline Axe 5 & 1,05 & 1,04 & R/R2 \\
\hline Axe 6 & 0,92 & 0,94 & R/R2 \\
\hline
\end{tabular}

3. Caractéristiques des deux roues et de leurs diffuseurs.

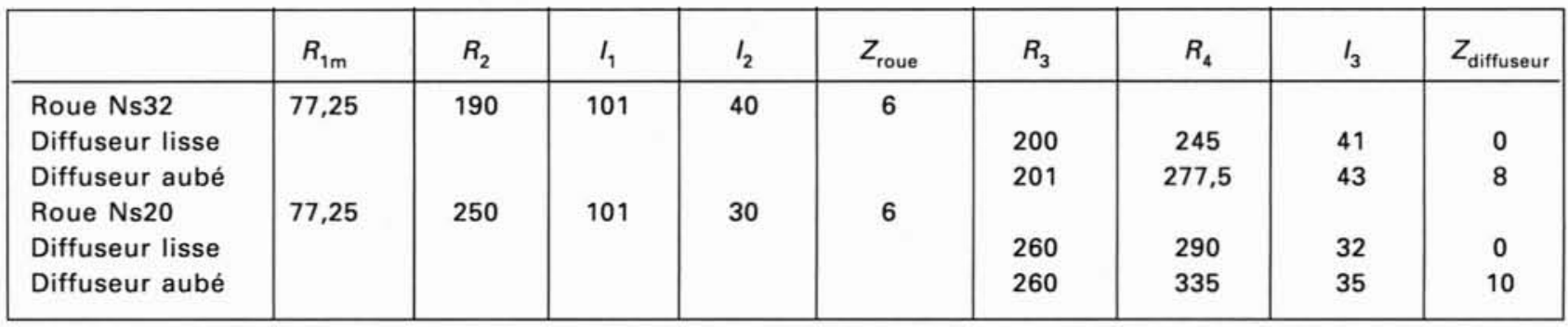

Tableau 1 : Caractéristiques des deux roues et de leurs diffuseurs.

devient fortement tridimensionnel en raison de la géométrie des roue, diffuseur et volute.

Dans les canaux de la roue, l'écoulement relatif dans le repère lié à celle-ci est stationnaire lorsqu'elle fonctionne avec le diffuseur lisse. Dans le cas de fonctionnement avec diffuseur aubé, il reste quasi-stationnaire sauf en très proche sortie à cause de la présence en aval, des bords d'attaque des aubages du diffuseur. L'écoulement sortant de la roue conserve bien évidemment ses caractéristiques de périodicité, de turbulence et d'instationnarité. Seul le caractère tridimensionnel a perdu un peu de son intensité du fait de la géométrie radiale de cette partie de la roue. Lors des fonctionnements hors régime nominal, à cause de l'inadaptation flux-profil, des fluctuations locales très importantes de vitesses viennent accentuer et amplifier les niveaux de turbulence et d'instationnarité de l'écoulement dans cette partie de la machine.

Nous avons déjà montré [1] et [2] que l'influence du diffuseur à aubes sur l'écoulement dans les canaux de la roue ne se faisait sentir qu'en proche sortie. Les résultats que nous présentons ci-après sont donc volontairement limités aux mesures de vitesses relevées en sortie roue et entrée diffuseur (lisse ou à aubes). Les fichiers comportent, pour chaque point de mesure, la position angulaire de la roue par rapport au diffuseur, et deux composantes du vecteur vitesse, $V d$ et $V u$ (voir fig. 2). Nous admettrons que les fluctuations de la composante de vitesse suivant l'axe $z$ s'effectuent autour d'une moyenne nulle, ce qui revient à considérer que l'écoulement moyen dans cette partie radiale de la machine est une superposition d'écoulements bidimensionnels. Une des manières d'aborder l'étude des écoulements turbulents en turbomachines consiste à décomposer le mouvement instantané en «mouvement moyen " et " mouvement turbulent » et d'écrire, par exemple pour la vitesse instantanée :

$$
V_{\text {instantance }}=\langle V\rangle+V^{\prime}
$$

La vitesse $\langle V\rangle$ est la moyenne de phase, correspondant à la somme d'une moyenne temporelle $(V$ : valeur constante dans le temps pour un régime établi) et d'une composante périodique.

Pour une position angulaire $\theta$ de la roue, la moyenne de phase en un point $M$ quelconque de l'écoulement sera alors :

$\left\langle V_{(M)}\right\rangle_{\theta}=\frac{1}{\Delta \theta} \int_{\theta-\frac{\Delta \theta}{2}}^{\theta+\frac{\Delta \theta}{2}} V_{(M, t)} d t=\frac{\sum_{i=1}^{i=n} V_{(M)_{\theta}}}{n}$

Les moyenne de phase et moyenne temporelle représentées sur la figure 4 sont ainsi extraites du fichier des mesures brutes de vitesse obtenues par vélocimétrie laser.

Le taux de turbulence est calculé, pour chaque valeur de $\theta$ en définissant :

$\rightarrow$ l'écart type, qui représente le carré de l'intensité de turbulence :

$\sigma_{\theta}=\sqrt{\left\langle\left(V_{\theta}^{\prime}\right)^{2}\right\rangle}=\sqrt{\frac{\sum_{i=1}^{i=n}\left[V_{\theta}-\left\langle V_{\theta}\right]^{2}\right.}{n}}$ 
$\rightarrow$ le taux de turbulence :

$\tau_{\theta}=\frac{\sigma_{\theta}}{\langle\rangle_{\theta}} \times 100=\frac{\sqrt{\frac{1}{n} \sum_{i=1}^{i=n}\left[V_{\theta}-\left\langle V_{\theta}\right]^{2}\right.}}{\frac{1}{n} \sum_{i=1}^{i=n}\left[V_{\theta}\right]} \times 100$

Pour les axes 5 (diffuseurs) et 6 (roues), toutes les grandeurs définies précédemment ont été systématiquement calculées, pour chaque valeur du débit expérimentée. Avant d'analyser les niveaux de turbulence mesurés, il faut bien voir que la

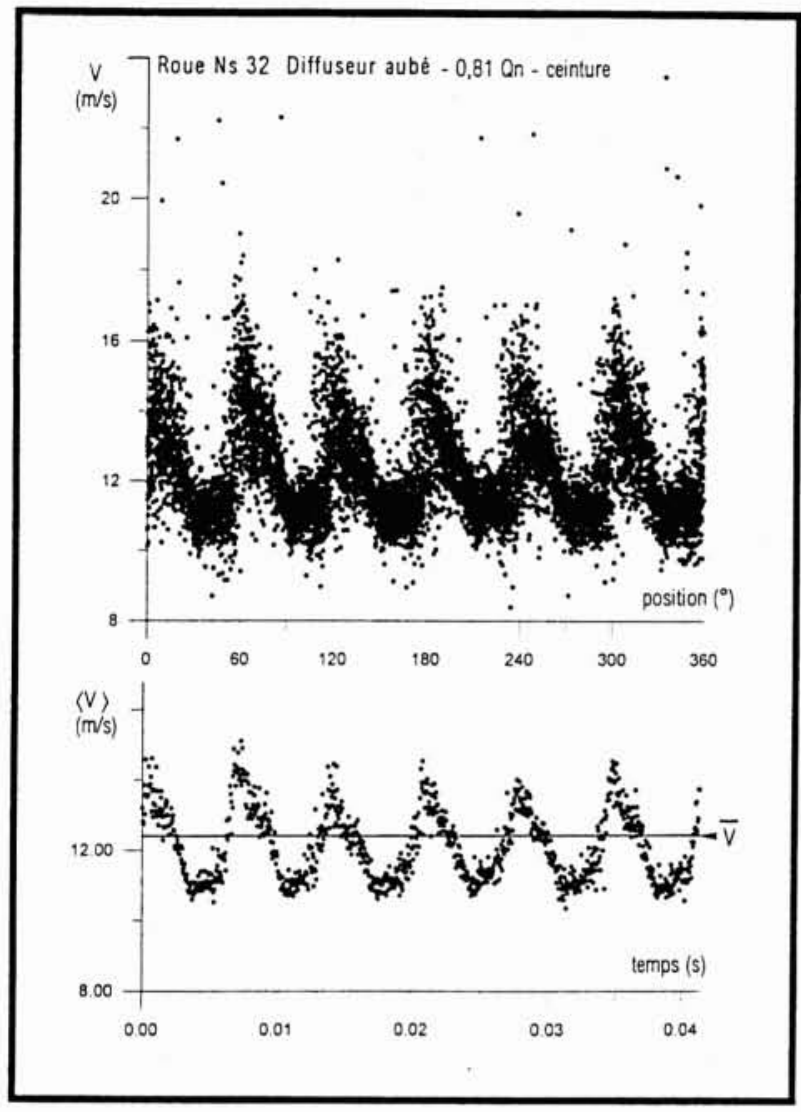

4. Moyenne de phase et moyenne temporelle. turbulence en environnement turbomachine, est le résultat de la nature «purement " turbulente de l'écoulement à laquelle s'ajoutent des fluctuations de vitesses de nature périodique. Dans le diffuseur aubé, cette turbulence intègre également, en plus de tous les phénomènes générés par la roue présents en fonctionnement diffuseur lisse, des fluctuations de vitesses importantes dues aux chocs se produisant aux bords d'attaque des aubes lorsque l'inadaptation d'incidence devient importante.

\subsection{Analyse des résultats}

\subsubsection{Fluctuations périodiques}

La figure 5 représente les moyennes de phase de la vitesse, obtenues en sortie des roues Ns20 et Ns32 munies de diffuseurs lisses et aubés (débit nominal - axe $5 \mathrm{c}-$ milieu du canal). Cet exemple révèle certains des aspects de l'influence du diffuseur aubé sur l'écoulement en sortie de roue. On peut notamment observer l'apparition d'une perturbation supplémentaire, à la fréquence de passage des aubes de la roue devant une aube de diffuseur.

Sur la figure 6, sont reportées en fonction du débit les évolutions des fluctuations périodiques mesurées dans le plan médian de la pompe (à mi-hauteur des aubages) à l'entrée des diffuseurs : axe 5 seul, pour les diffuseurs lisses, moyenne des axes $5 \mathrm{a}, 5 \mathrm{~b}$ et $5 \mathrm{c}$ pour les diffuseurs à aubes.

Le taux de fluctuation (en \%) est défini par:

Taux de fluctuation $:=\frac{\left\langle\eta_{\max }-\langle\rangle_{\min }\right.}{\bar{V}}$

On constate sur ces figures des différences importantes de comportement entre la roue Ns20 et la roue Ns32. Les fluctuations périodiques, en l'absence de diffuseur aubé, évoluent peu en fonction du débit en sortie de roue Ns20 alors qu'elles augmentent avec le débit pour la roue Ns32. Cela est en étroite relation avec les différences d'évolutions constatées sur les champs moyens de vitesses produits par ces deux roues ([2] et [3]), notamment au niveau des zones de recirculation dans le diffuseur lisse.

La présence d'un diffuseur aubé gomme légèrement ces différences avec un minimum de fluctuations périodiques près du point d'adaptation (à $Q n$ pour la roue Ns20 et 0,8 Qn pour la roue Ns32), et un maximum à débit partiel ( $0,4 Q n$ environ pour chacune des roues). Les diffuseurs pos-

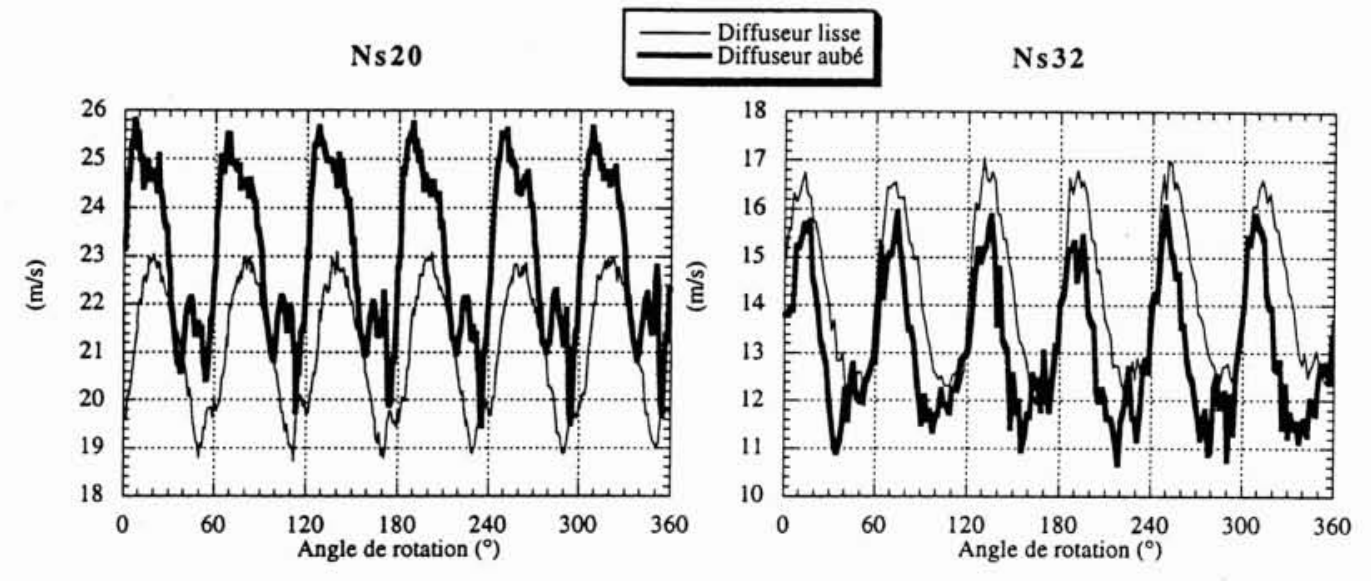

5. Exemple de moyenne de phase en sortie roue. 


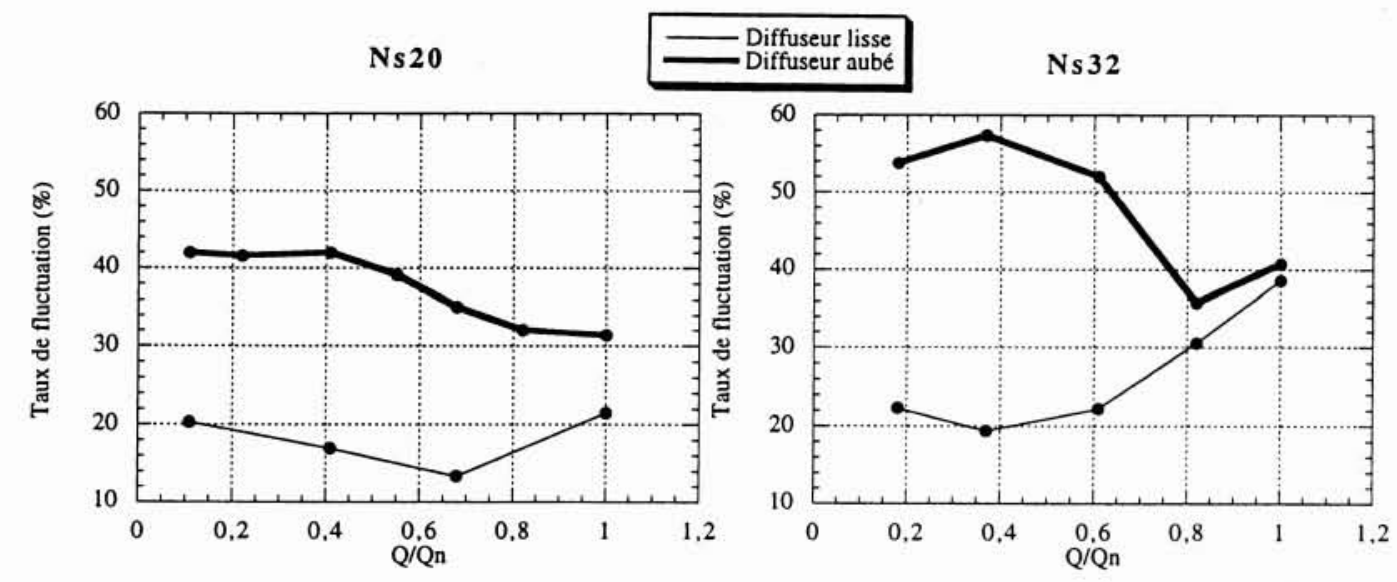

6. Evolution des fluctuations périodiques.

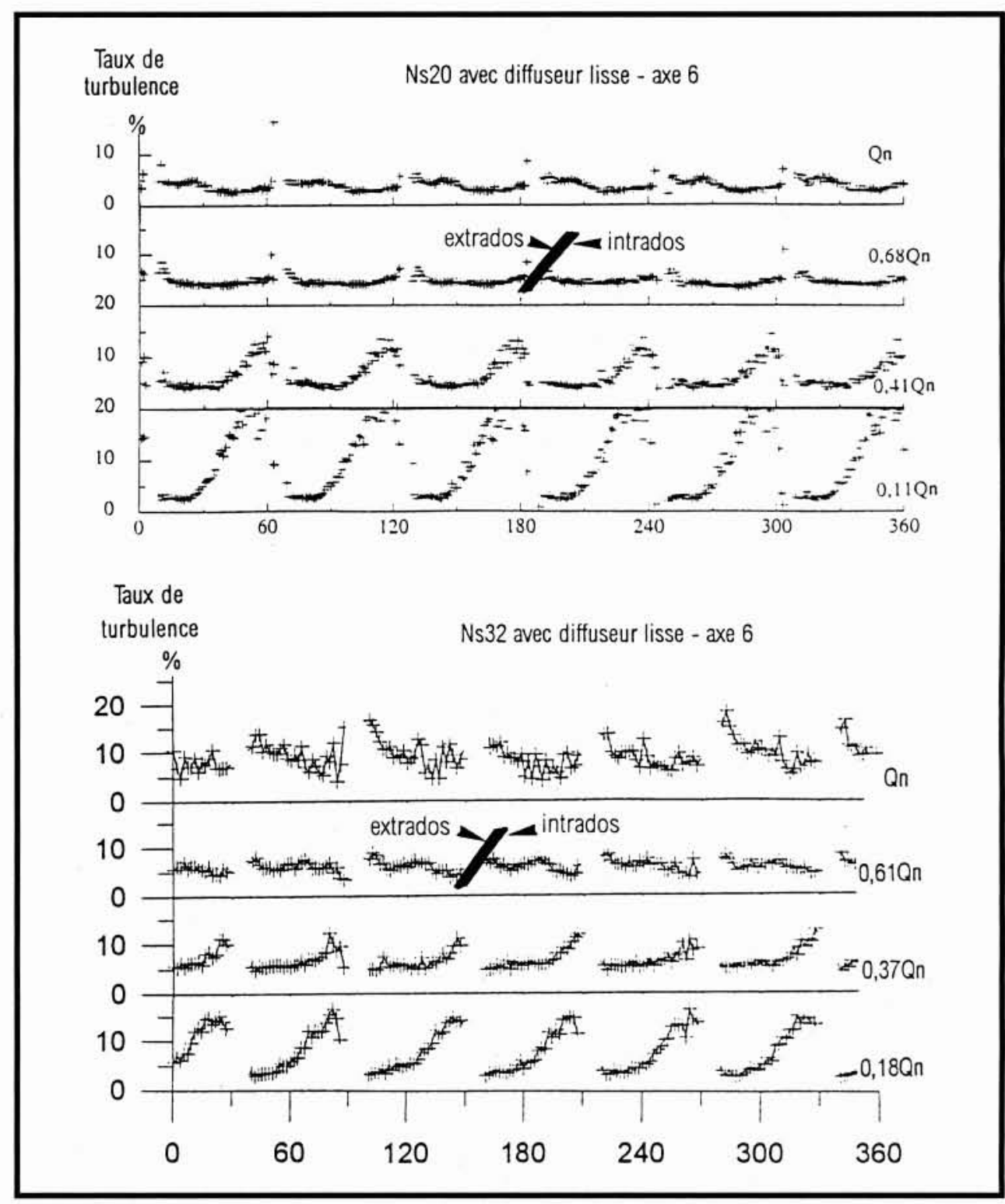

7. Evolution de la turbulence en fonction du débit dans les roues NS20 et NS32. 
sédant 10 aubes pour la pompe Ns20 et 8 pour la Ns32, on confirme ici la nette influence de ce nombre d'aubes sur le taux de fluctuations périodiques avec un taux plus faible sur la Ns20. On peut rapprocher ces travaux de ceux des auteurs référencés [4] et [5]. Le bruit généré par cette interaction roue-diffuseur restera cependant moins élevé sur la roue Ns32 munie de son diffuseur aubé avec, en niveau RMS moyen au débit nominal, une fluctuation de vitesse de l'ordre de $4 \mathrm{~m} / \mathrm{s}$ alors qu'en sortie de la roue $\mathrm{Ns} 20$ ce niveau atteint $5 \mathrm{~m} / \mathrm{s}$. A $0,4 Q n$ les bruits seront voisins, avec des niveaux RMS proches de $7,5 \mathrm{~m} / \mathrm{s}$ dans les deux cas.

\subsubsection{Analyse de la turbulence}

\section{A) Dans les roues}

Les niveaux de turbulence mesurés dans les roues restent quasiment identiques, que celles-ci soient suivies d'un diffuseur lisse ou à aubes. Sur l'exemple de la figure 7 , on constate que le taux de turbulence maximal se déplace de l'intrados (face concave) à l'extrados (face convexe) lorsque le débit décroît. Cette évolution est due au basculement progressif des lignes de courant dans les canaux de la roue ainsi qu'aux décollements pariétaux qui en résultent.

\section{B) Dans les diffuseurs}

Sur la figure 8 ont été reportés les taux de turbulence en sortie de chacune des roues (axe 5 dans les diffuseurs lisses et axe $5 \mathrm{a}$ proche du bord d'attaque d'une aube dans les diffuseurs aubés). Les résultats obtenus avec et sans diffuseurs aubés, sont présentés pour deux débits de fonctionnement éloignés. Dans le diffuseur aubé on distingue, au débit le plus élevé, une périodicité au sein de l'évolution de la turbulence sur un tour de roue. Cette allure, qui n'apparaît pas avec diffuseur lisse, est due aux passages successifs des aubes de la roue devant le bord d'attaque de l'aube du diffuseur proche du point de mesure.

Pour ce même débit, les taux de turbulence sont en moyenne plus faibles en sortie de roue Ns20 et l'évolution périodique est moins marquée que pour la Ns32. Pour les deux roues la présence du diffuseur aubé a pour effet de diminuer les niveaux de turbulence. Cela reste vrai pour la roue $\mathrm{Ns} 20$ à débit très faible alors qu'en sortie de roue Ns32 le taux augmente plus vite dans le diffuseur aubé que dans le diffuseur lisse. Cette dernière constation est reproduite sur la figure 9 où sont reportés, en fonction du débit, les taux de turbulence mesurés dans la roue $\mathrm{Ns} 32$ avec diffuseur lisse et diffuseur aubé.

Cette figure 9 permet de constater :

1) Dans la roue : que le taux de turbulence est inchangé lorsque l'on passe du fonctionnement avec diffuseur lisse à celui avec diffuseur aubé, pourtant générateur d'instationnarités. La frontière partie tournante/partie fixe semble difficilement franchissable, non seulement par les écoulements de retour, comme nous l'avons déjà montré, mais également pour les fluctuations locales de la vitesse.

2) Dans le diffuseur lisse : que la turbulence suit une allure identique (en fonction du débit) à celle relevée dans la roue. Le taux de turbulence est toutefois deux fois plus important qu'en sortie roue, en raison du lâcher des sillages en provenance des aubes de roue dans cette partie fixe de la pompe et des décollements sur les parois du diffuseur.

3) Dans le diffuseur aubé : il semble que l'adaptation roue diffuseur soit effective entre 80 et $90 \%$ du débit nominal de la roue. C'est en effet pour ce débit que la turbulence est minimale, les fluctuations de vitesses sont donc faibles, ce qui implique une incidence à l'attaque des aubages du diffuseur également très faible. En s'éloignant de ce régime, on note que le taux de turbulence augmente rapidement, en raison de la désadaptation des aubages du diffuseur à l'écoulement sortant de la roue.

\section{IV —CONCLUSIONS}

Les phénomènes d'écoulements instationnaires dans une pompe centrifuge sont explicités dans ce document en comparant deux configurations de diffuseurs (lisse et aubé) pour deux roues de géométries différentes (Ns20 et Ns32). Décrits

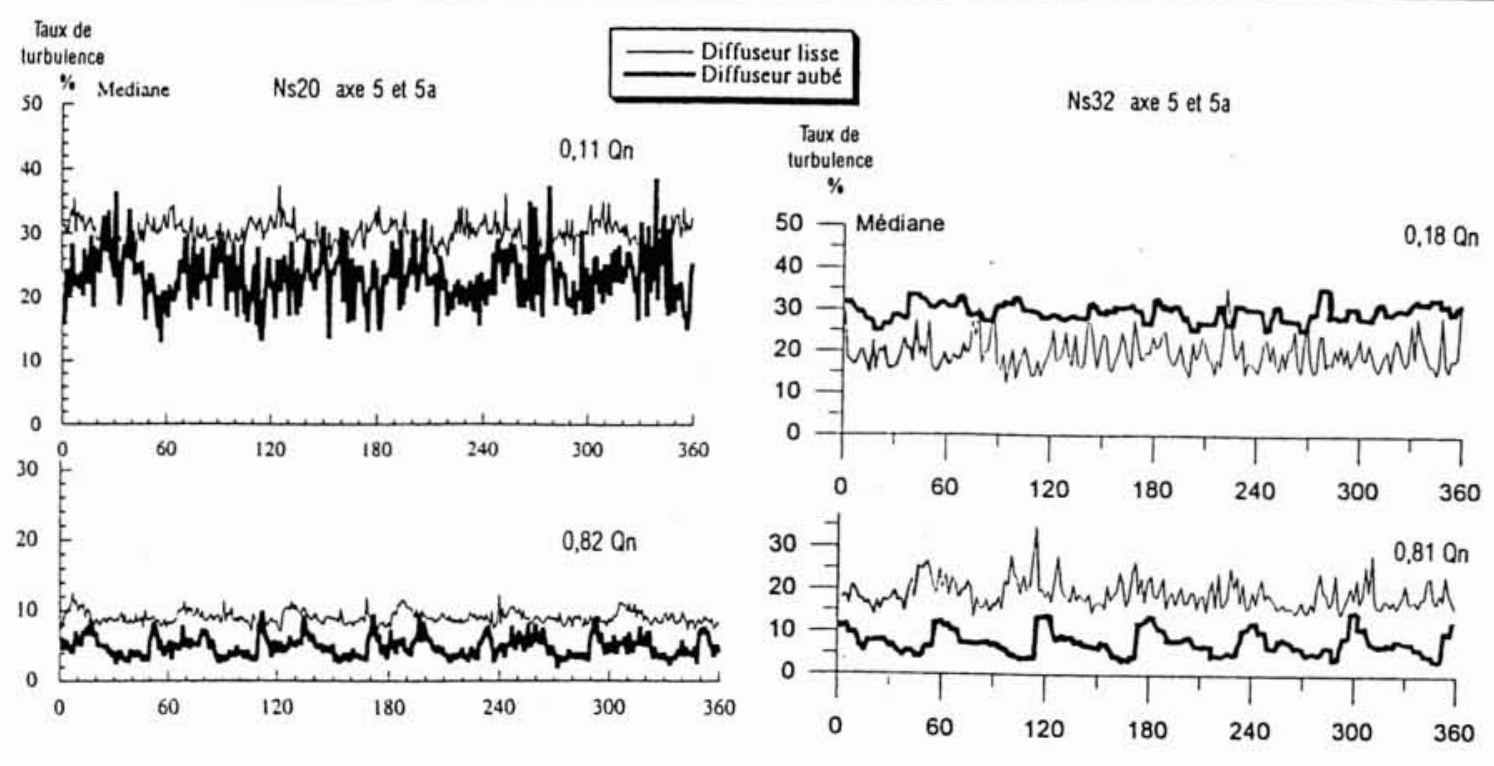

8. Evolution de la turbulence dans les diffuseurs. 


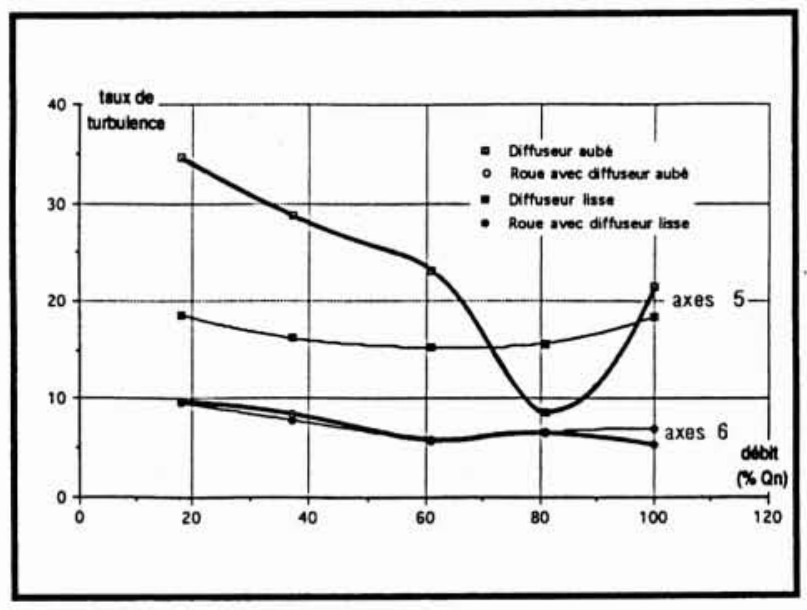

9. Taux de turbulence en fonction du débit.

à l'aide de la vélocimétrie laser, les mouvements périodiques et turbulents du fluide ont pu être analysés sur une large plage de fonctionnement de la pompe.

Cette contribution à l'étude des écoulements dans les pompes confirme que la frontière est forte entre le mobile et son environnement extérieur fixe. On a ainsi pu observer que la nature de la turbulence à l'intérieur des canaux de la roue n'était pas modifiée par la présence d'un diffuseur aubé et qu'il y avait une rapide perte d'informations sur cet écoulement relatif entre les aubes dès que le fluide qui sort de la roue se mélange à l'écoulement aval. Les fluctuations d'écoulement restent finalement très localisées, mais seront propagées sous forme d'ondes hydroacoustiques dans l'ensemble de la pompe et de son circuit. Du point de vue de l'adaptation roue/diffuseur aubé on a pu constater de profondes différences entre les deux pompes de vitesses spécifiques 20 et 32 :

$\rightarrow$ Les niveaux de fluctuations périodiques sont fortement influencés par la présence du diffuseur aubé dans la roue $\mathrm{Ns20}$, alors que cette influence est faible au point d'adaptation de la Ns32.

$\rightarrow$ Les niveaux de turbulence, alors qu'ils ont un comportement similaire dans les deux roues, sont profondément modifiés une fois mesurés dans les diffuseurs.
Le résultat le plus marquant est obtenu au débit d'adaptation du diffuseur aubé pour la roue de Ns32, où l'on observe que la présence de cet organe supplémentaire dans la pompe conduit à un taux de fluctuations de vitesses en sortie de roue plus faible qu'en son absence. Les aubes du diffuseur introduisent cependant à leur tour des sillages tels que le bruit total généré par la pompe risque d'être plus élevé. Pour une conception plus silencieuse des pompes on sera donc conduit à optimiser l'adaptation de la roue au diffuseur aubé, mais aussi à limiter le bruit propre généré par ce dernier. Par ailleurs, l'influence de l'entrefer (jeu roue-diffuseur) qui est un paramètre essentiel dans l'étude des interactions rotor-stator reste à analyser.

\section{RÉFÉRENCES}

[1] Toussaint M., Hureau F. - « Analysis of flow measurements in the impeller and vaned diffuser of a centrifugal pump operating at part load $» . \mathrm{XVIII}^{\mathrm{e}} \mathrm{IAHR}$ Symposium on Hydraulic Machinery and Cavitation. Valencia, Spain, September 1996

[2] Toussaint M., Hureau F. - « Etude expérimentale de l'écoulement instationnaire dans une pompe centrifuge fonctionnant à débit partiel ». IAHR, 6th International Meeting. The behavior of hydraulic machinery under steady oscillatory conditions. Lausanne, September 1993.

[3] Hureau F., Kermarec J., Stoffel B., Weiss K. - « Study of internal recirculation in centrifugal impellers ». ASME, 2nd Pumping Machinery Symposium, Washington D.C June 20-24, FED Vol. 154, pp. 151-157.

[4] Casey M.V., Eisele K., Muggli F.A., Gulich J., SchaCHENMANN A. - « Flow analysis in a pump diffuser part 2 : Validation of a CFD Code for steady flow $»$. ASME, Numerical simulations in turbomachinery, Vol. 227 pp. 135 143.

[5] Arndt N., Acosta A.J., Brennen E., Caughey T.K. " Experimental Investigation of Rotor-Stator Interaction in Centrifugal Pump with Several Vaned Diffusers ». ASME, Journal of Turbomachinery, Vol. 112, January 1990. 